\title{
Highlights from this issue
}

doi:10.1136/gut.2011.242685

Luminal Gl

\section{Alcohol intake and oesophageal cancer}

Oesophageal squamous cell cancer (OSCC), oesophageal adenocarcinoma (OA) and oesophago-gastric junction adenocarcinoma (OGJA) are leading causes of death worldwide. While alcohol is an established cause of OSCC, its relationship to the other two subtypes is still unclear. In this landmark multi-national study, Freedman et al provide definitive answers. Pooling data from nine case control studies and two cohort studies of the Barrett's Oesophagus and Oesophageal Adenocarcinoma Consortium (BEACON), they were able to examine 1016 OSCC, 1821 OA, 1837 OGJA cases and 10854 controls. Heavy alcohol consumption (seven or more drinks per day) was not associated with increased risk of OA or OGJA. Modest alcohol consumption appeared to be associated with reduced risk for both types of cancer. In contrast, alcohol intake was strongly associated with increased risk of OSCC (OR for $\geq 7$ drinks per day $9.62,95 \%$ CI 4.26 to 21.71 ). The findings highlight the difference in aetiology between the subtypes of oesophageal cancer and will aid clinicians in offering appropriate advice about the causes of cancer to their patients (see page 1029).

\section{Targeting MAdCAM in ulcerative colitis}

Biological therapy has transformed management of IBD but more strategies are still needed. Homing of leucocytes to the gut mucosa is a promising target for therapeutic intervention in IBD shown by studies with the $\alpha 4$-integrin antibody natalizumab. However, natalizumab has a risk of progressive multifocal leucoencephalopathy. To avoid this, more selective strategies targeting the $\alpha 4 \beta 7 / \mathrm{MAdCAM}$ pathway in the gut would retain the efficacy of natalizumab but offer a better safety profile. In this issue of Gut, Vermeire et al report the first-in-human study of a monoclonal antibody to MAdCAM, PF-00547,659, aimed to explore the safety and preliminary efficacy of this gut-specific mechanism in ulcerative colitis. In this randomised, double-blind placebocontrolled study, 80 patients with active ulcerative colitis received single or multiple (three doses, 4-week intervals) doses of

Emad El-Omar, Alexander Gerbes and William Grady, Editor and Deputy Editors

PF-00547, 659 0.03-10 mg/kg IV/SC, or placebo. The results show that single and multiple doses of PF-00547, 659 were safe and well tolerated with no evidence of immunogenicity. Preliminary efficacy findings suggest higher response and remission rates for PF-00547,659 as compared to placebo. The authors suggest that PF-00547, 659 is a promising target for large phase II studies in ulcerative colitis (see page 1068)

\section{Where you get your cancer care matters}

In this issue of Gut, Morris and colleagues report on their study of death rates in patients with colorectal cancer in Norway, Sweden, and England. They identified people diagnosed with colorectal cancer between 1996 and 2004 in a population based study and measured the 5-year cumulative relative period of survival and excess death rates stratified by age and period of follow-up in order to examine the relative survival and excess death rates of patients with colorectal cancer in Norway, Sweden and England. Notably, they found survival of English patients with colorectal cancer was significantly lower than was observed in both Norway and Sweden. Five-year age-standardised colon cancer relative survival was $51 \%$ in England versus 58\% in Norway and 60\% in Sweden. Five-year rectal cancer survival

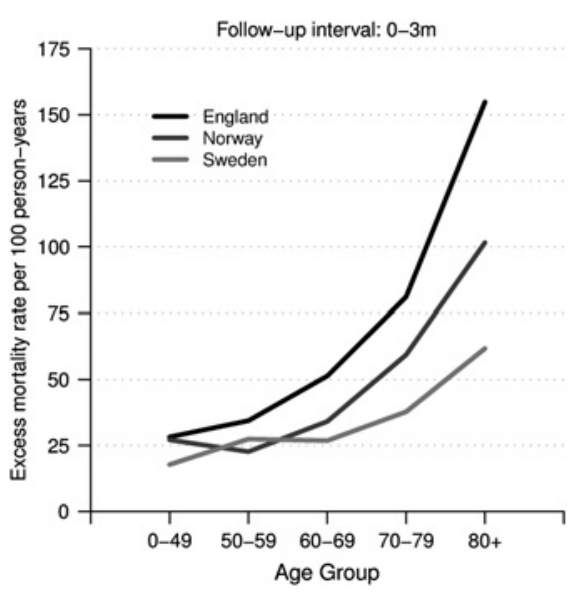

Figure 1 Excess death rates among colon cancer patients in England, Norway and Sweden (2001-2004) by age at diagnosis and period of follow-up. was $52 \%$ in England versus $61 \%$ and $60 \%$ in Norway and Sweden, respectively. The authors suggest that the lower survival for colon cancer in England was primarily due to a high number of excess deaths among older patients in the first 3 months after diagnosis, possibly because a greater proportion of the population in England present with more rapidly fatal disease (especially in the older age groups) than in Norway or Sweden. These results obviously raise significant questions about colorectal cancer care in the $\mathrm{EU}$ and suggest further assessment of how this care is delivered in England versus Norway and Sweden (see page 1087).

\section{One more reason to have a glass of red wine}

The metabolic syndrome has been associated with an increased risk of a variety of diseases including colorectal cancer, but only a few studies have assessed the dyslipidaemia component of the metabolic syndrome in relation to the risk of colorectal cancer. Duijnhoven and coworkers have now examined the association between serum concentrations of total cholesterol, high density lipoprotein

Table 4 Additional adjustments for association between HDL-cholesterol and colon cancer risk

\begin{tabular}{|c|c|}
\hline Model & $\begin{array}{l}\text { Continuous } \uparrow \\
\text { Per } 1 \text { SD increase }\end{array}$ \\
\hline Adjusted* & $0.78(0.68$ to 0.89$)$ \\
\hline Adjusted $\dagger$ & 0.77 (0.61 to 0.97$)$ \\
\hline Adjusted ++ all biomarkers $\neq$ & 0.80 (0.62 to 1.02$)$ \\
\hline $\begin{array}{l}\text { Adjusted } \dagger+4 \text { factors metabolic } \\
\text { syndrome } \S\end{array}$ & 0.75 (0.59 to 0.97$)$ \\
\hline $\begin{array}{l}\text { Adjusted } \dagger+\text { all biomarkers } \ddagger \\
+4 \text { factors metabolic syndrome } \S\end{array}$ & $0.77(0.59$ to 1.00$)$ \\
\hline
\end{tabular}

${ }^{*}$ Conditioned on matching factors and adjusted for height, weight, smoking habits, physical activity, education, consumption of fruit, vegetables, meats, fish and alcohol, intake of fibre, energy from fat and energy from non-fat; $\mathrm{N}$ cases/controls $=612 / 612$.

†Conditioned on matching factors and adjusted for height, weight, smoking habits, physical activity, education, consumption of fruit, vegetables, meats, fish, and alcohol, intake of fibre, energy from fat and energy from non-fat;

After removing all cases and controls with missing data for the additional confounders; $\mathrm{N}$ cases/controls $=235 / 235$.

‡Included in 'all biomarkers' are blood concentrations of $\mathrm{C}$ reactive protein, glycosylated haemoglobin, $\mathrm{C}$ peptide, and Reactive Oxygen Metabolites.

SIncluded in 'four factors metabolic syndrome' are waist circumference, triglycerides, blood pressure and diabetes mellitus.

qIncreases in one SD in HDL-cholesterol were $16.6 \mathrm{mg} / \mathrm{dl}$. 
cholesterol (HDL), low density lipoprotein cholesterol, triglycerides, apolipoprotein A-I (apoA), apolipoprotein B and the incidence of colorectal cancer (CRC). They used a nested case-control study design to examine subjects enrolled within the European Prospective Investigation into Cancer and Nutrition cohort, which has more than 520000 participants from 10 western European countries. They found that the concentrations of $\mathrm{HDL}$ and apoA were inversely associated with the risk of colon cancer although no association was observed with the risk of rectal cancer. Importantly, additional adjustment for biomarkers of systemic inflammation, insulin resistance and oxidative stress did not influence the association between HDL and risk of colon cancer. If confirmed, this may be one more reason to have a glass of red wine with your evening meal (see page 1094)

\section{Hepatology}

Novel aspects in portal hypertension Von Willebrand factor (vWF) as independent predictor of prognosis

Endothelial dysfunction is increasingly being considered as a determinant of portal hypertension. This interesting study from Barcelona investigated vWF and other endothelial markers in patients with mild to moderate cirrhosis. They observed a correlation between peripheral

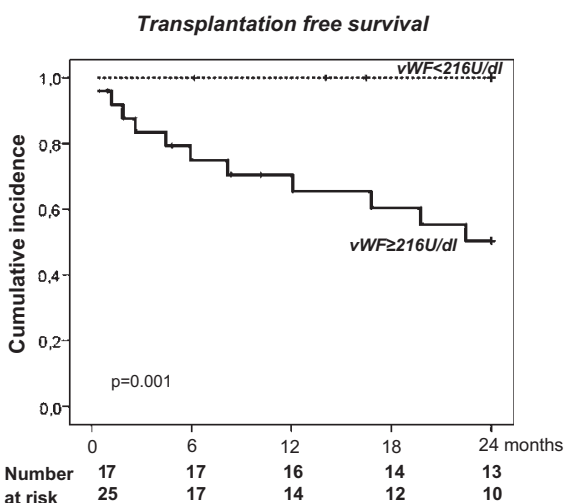

Figure 3B Transplantation-free survival (11 events) in patients with cirrhosis and vWF levels above and below $216 \mathrm{UI} / \mathrm{dl}$. *HR indicating the risk of the event adjusting for portal hypertension and liver function.

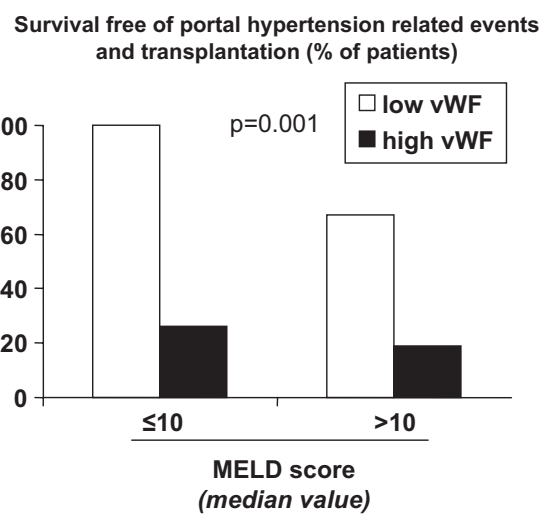

Figure 4 Patients with high vWF had a poor outcome independently from the MELD.

venous vWF concentrations and the degree of portal hypertension. Moreover, during follow-up complications of portal hypertension were more frequent and transplant-free survival was lower (figure 3B) in patients with high vWF concentrations. Importantly, this was observed in patients with low and with higher
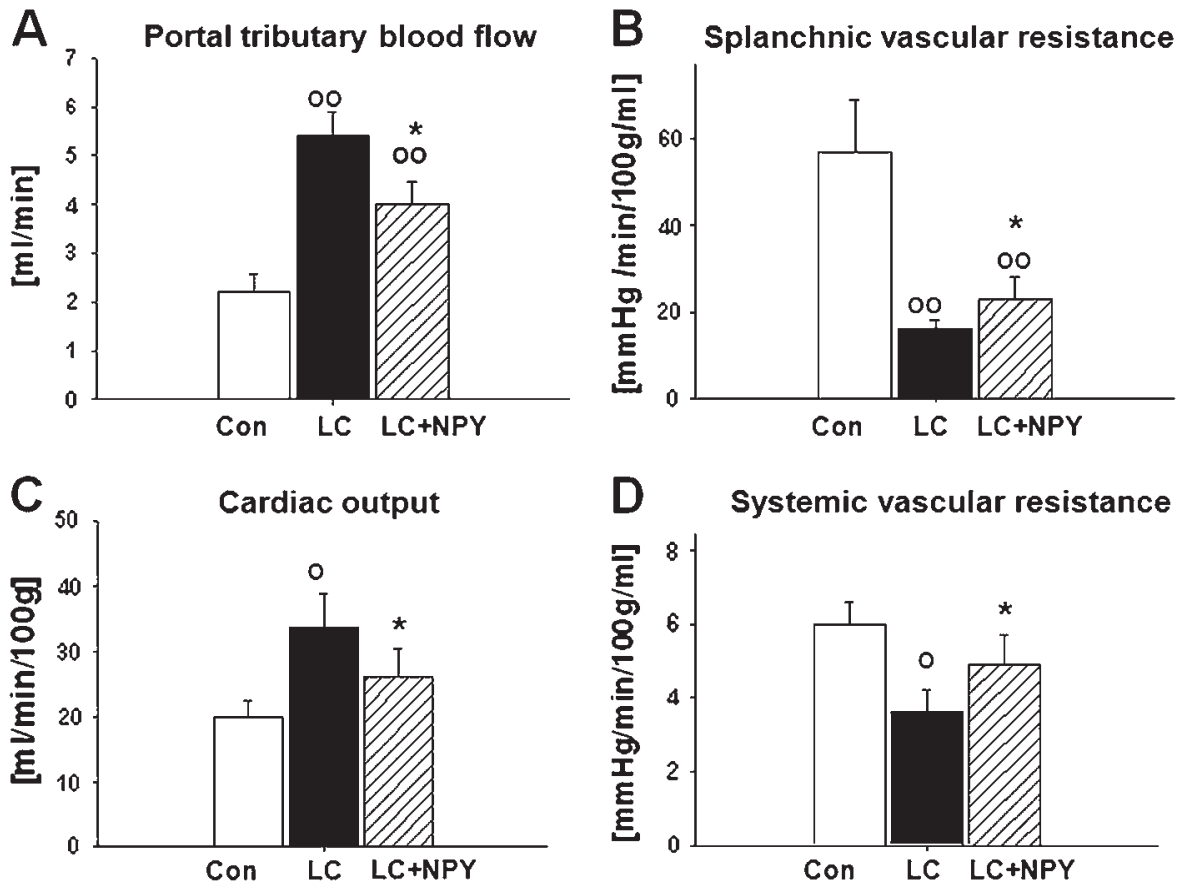

Figure 2 Portal tributary flow (A), splanchnic vascular resistance (B), cardiac output (C) and systemic vascular resistance (D) in control $(n=7)$ and cirrhotic ascitic rats $(L C, n=8)$ after acute intravenous administration of NPY. ${ }^{*}: p<0.05$ versus $L C, 0: p<0.05$ and $00: p<0.01$ versus control. LC, liver cirrhosis.

MELD score, respectively (figure 4). These interesting findings should be investigated in an independent cohort including patients with more advanced cirrhosis (see page 1133).

\section{Neuropeptide Y (NPY): a novel} therapeutic agent for portal hypertension?

NPY, a sympathetic neurotransmitter, has recently received attention in portal hypertension. $\mathrm{R}$ Wiest and colleagues from Bonn and Regensburg in their interesting paper suggest a potential therapeutic role for NPY in a rat model of cirrhosis. Acute intravenous NPY administration reduced portal pressure in cirrhosis without affecting systemic blood pressure (figure 2). In further experiments they elegantly show that these effects may be due to inhibition of vascular $\mathrm{NO}$ production and stimulation of Rho-kinase signalling. Data on the effects of NPY in patients with cirrhosis are eagerly awaited (see page 1122). 